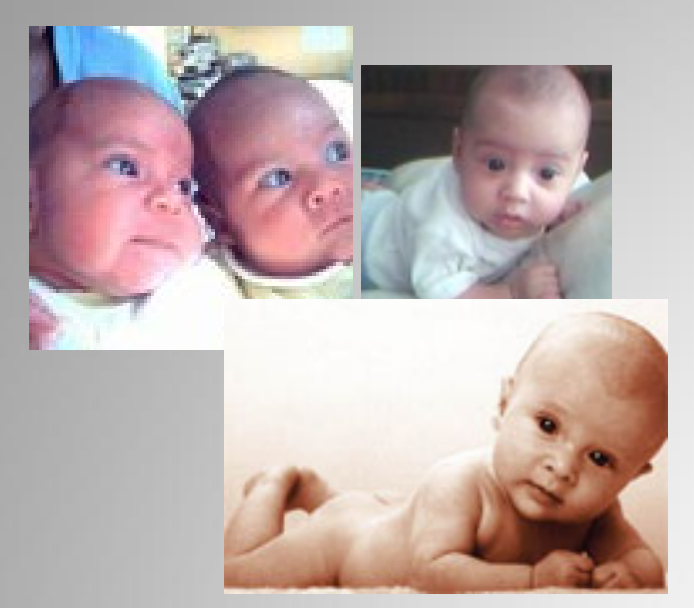

\title{
LA NATALIDAD y LA FECUNDIDAD
}

\section{Dra. Ana María Foschiatti}

Publicado en formato digital: Foschiatti, Ana María. LA NATALIDAD y LA FECUNDIDAD. Resúmenes. Revista Geográfica Digital. IGUNNE. Facultad de Humanidades. UNNE. Año 7. № 13. Enero - Junio 2010. Resistencia, Chaco. En: http://hum.unne.edu.ar/revistas/geoweb/default.htm 


\section{NATALIDAD}

La natalidad mide el número de nacimientos que se producen en un área y un tiempo, normalmente un año. Para estudiarla se utiliza la tasa de natalidad, que se expresa en proporción (por mil -\%o).

\section{MEDICIÓN DE LA NATALIDAD

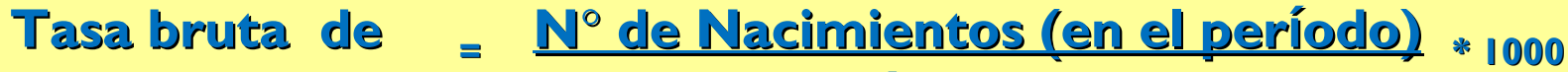 Natalidad (TN) Población Media x 1000}
ALTA si está por encima de $30 \%$
MODERADA entre 15 y $30 \%$
BAJA
por debajo del $15 \%$ 


\section{Distribución geográfica de la natalidad.}

Según las tasas de natalidad de los distintos países (2000) se pueden delimitar tres zonas diferentes en el mundo:

a) Países con tasas de natalidad altas (más del $40 \%$ \%): son aquellos países de más bajo desarrollo económico, entre los que podemos citar los del África Negra y algunos asiáticos.

b) Países con tasas de natalidad intermedias (entre el 20 y el $40 \%$ ): Son aquellos países que han iniciado la reducción de la natalidad pero que aun no han logrado estabilizarlas. Por lo general son los países en vías de desarrollo: Gran parte de Asia, el Norte de África y Latinoamérica.

c) Países con tasas de natalidad bajas (menores del $20 \%$ \%): Son los países desarrollados, que iniciaron pronto el descenso de la natalidad y hoy se encuentran con tasas estabilizadas, con ligeros altibajos coyunturales, $y$ con tasas que no aseguran la reposición generacional. Los países con menor natalidad son los del Sur y Este de Europa. 


\section{NATALIDAD (Estimación de Population Reference Bureau Inc.)}

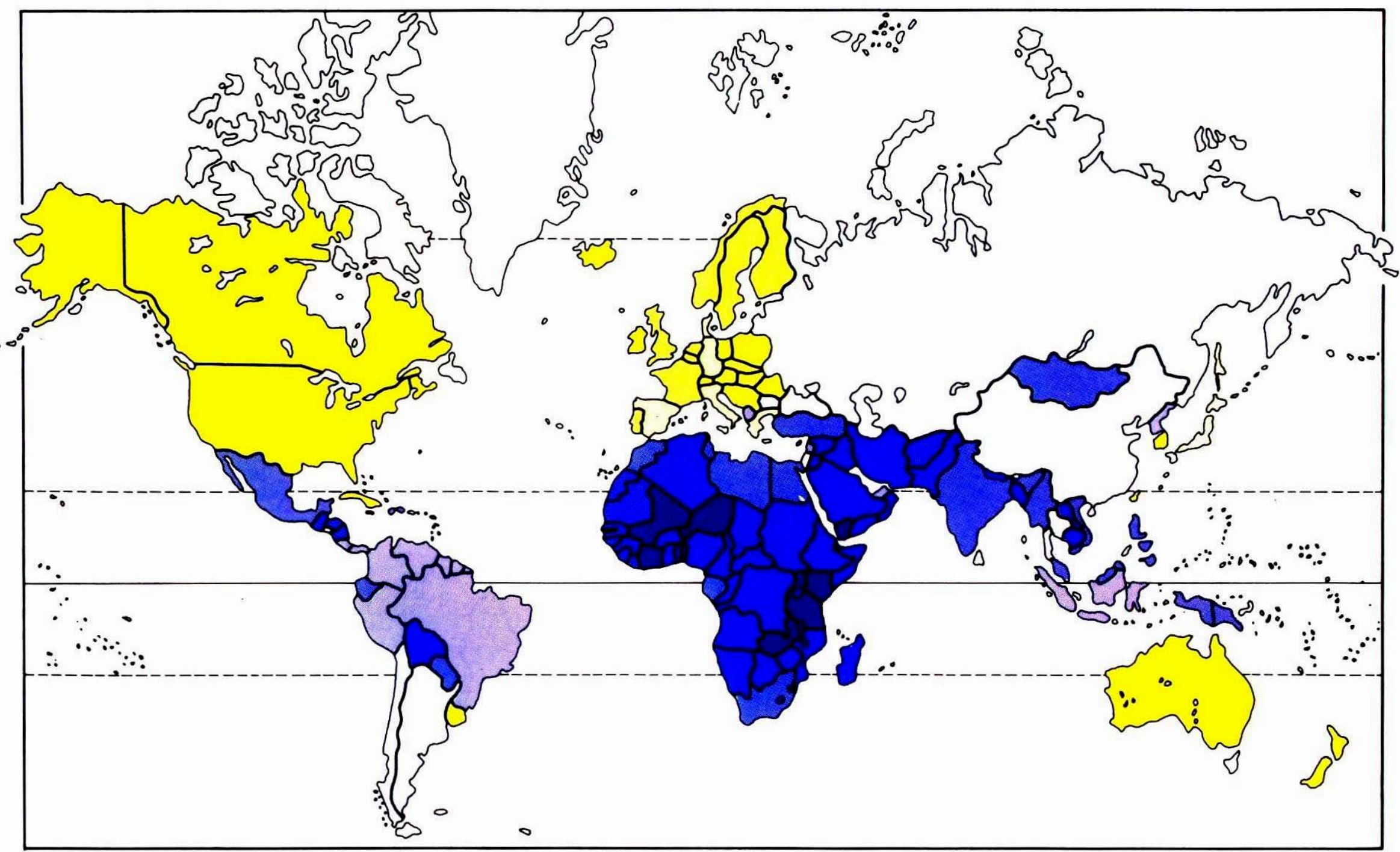

Menos de $12 \%$

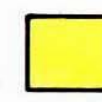

De 12 a $14,9 \%$

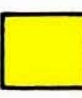

De 15 a $19,9 \%$

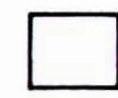

De 20 a $24,9 \%$ 。
De 25 a $29,9 \%$
De 30 a $39,9 \%$
De 40 a $49,9 \%$
$\mathrm{De}+\mathrm{de}$ $50 \%$ o

Publicado en formato digital: Foschiatti, Ana María. LA NATALIDAD y LA FECUNDIDAD. Resúmenes. Revista Geográfica Digital. IGUNNE. Facultad de Humanidades. UNNE. Año 7. № 13. Enero - Junio 2010. Resistencia, Chaco. En: http://hum.unne.edu.ar/revistas/geoweb/default.htm 


\section{Factores condicionantes de la natalidad.}

a) Económicos: desarrollo económico

b) Biológicos: población joven, nupcialidad temprana

c) Sociales: nivel económico y formación cultural d) Ideológicos: cuestiones religiosas y políticas 


\section{Evolución histórica de la natalidad. Consecuencias del descenso de la natalidad.}

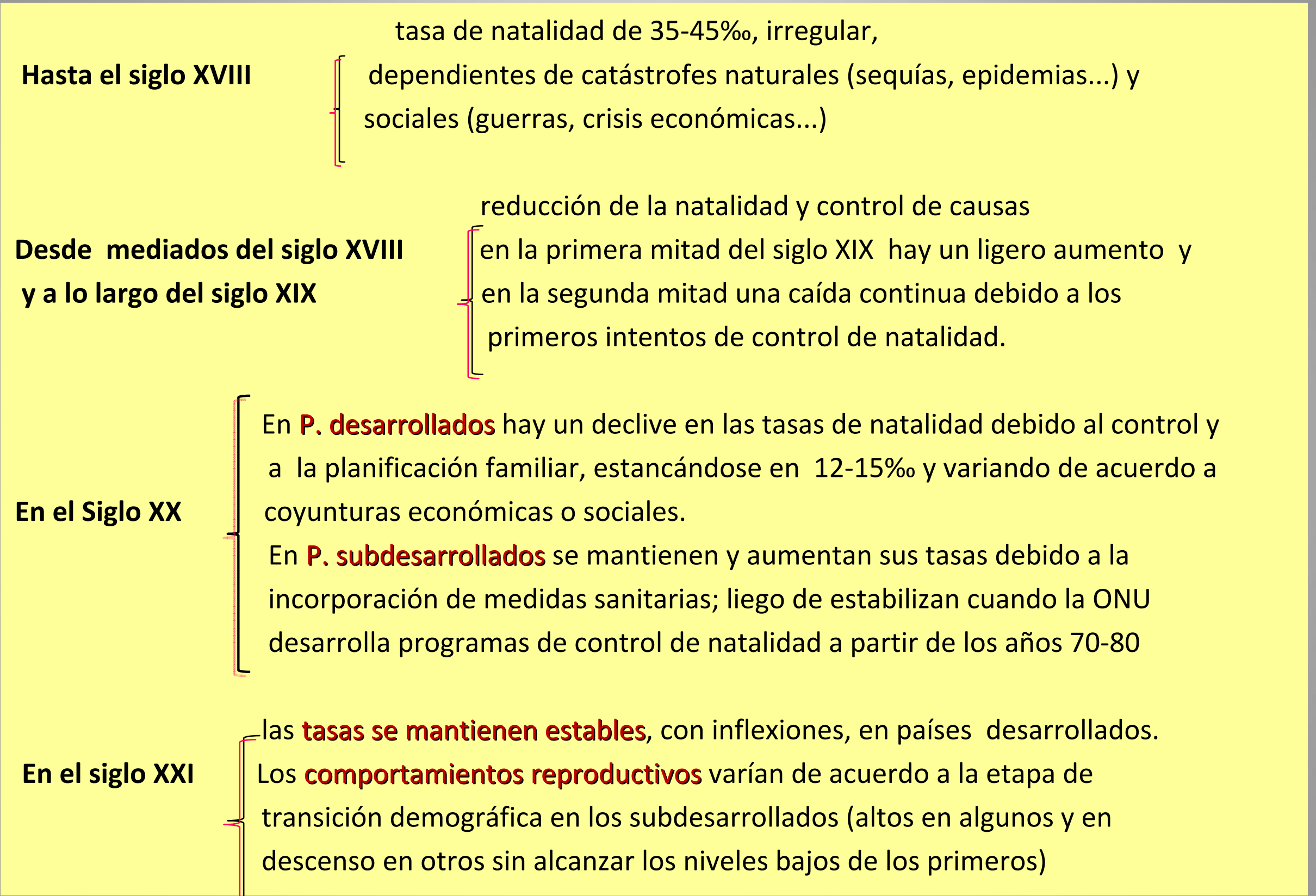




\section{Evolución de las Tasas de Natalidad en los Países Desarrollados}

\section{Tasa de Natalidad}

(\%)

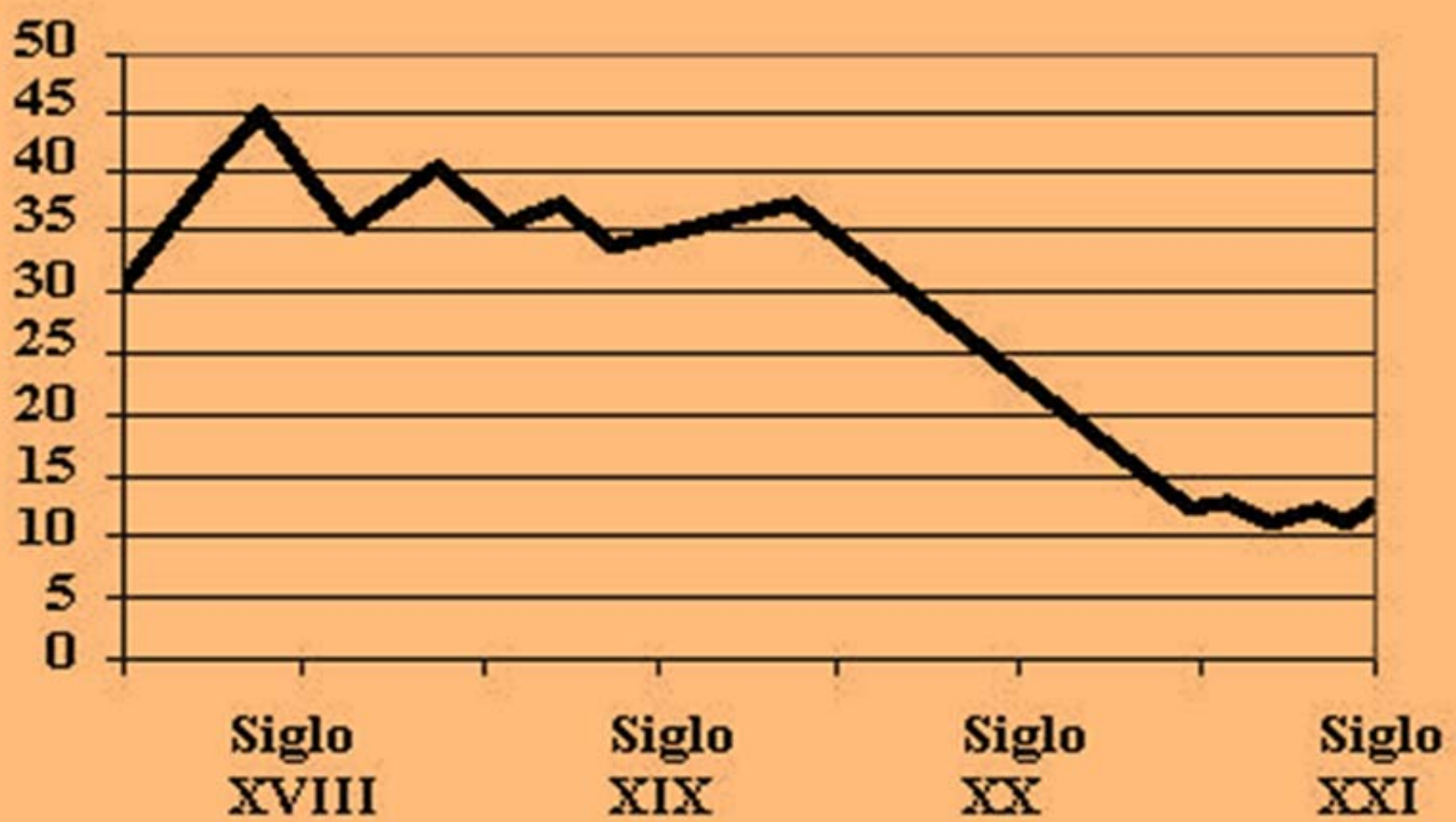

Publicado en formato digital: Foschiatti, Ana María. LA NATALIDAD y LA FECUNDIDAD. Resúmenes. Revista Geográfica Digital. IGUNNE. Facultad de Humanidades. UNNE. Año 7. № 13. Enero - Junio 2010. Resistencia, Chaco. En: http://hum.unne.edu.ar/revistas/geoweb/default.htm 


\section{FECUNDIDAD}

- Natalidad: Representa la cantidad de nacimientos ocurridos en toda la población.

- Edad Fértil: Período de tiempo donde está propensa a quedar embarazada. Este período se inicia aproximadamente con la menarquia a los 15 años y finaliza con la menopausia a los 50. Obviamente este patrón no es rígido para todas las mujeres.

- Fecundidad: Capacidad efectiva de una mujer, un hombre o una pareja, de producir un nacimiento. Se refiere a la cantidad de hijos que tiene una mujer durante su vida fértil.

- Fecundidad conyugal: Cantidad de hijos que una pareja concibe durante la unión o matrimonio. 
- Fecundidad natural: Fecundidad espontánea, no interviene ningún elemento externo o interno para disminuir su magnitud.

- Fecundidad controlada: se produce cuando la mujer, el hombre o la pareja, involucra elementos o comportamientos voluntarios tendientes a disminuir la magnitud de la fecundidad.

- Fertilidad: Capacidad biológica de una mujer, un hombre o una pareja de concebir un hijo.

(Se debe incluir la observación de que en el idioma inglés los conceptos de fecundidad y fertilidad están invertidos: fertility corresponde a fecundidad y fecundity corresponde a fertilidad)

- Fecundabilidad: Probabilidad que tiene una mujer de concebir durante un ciclo menstrual.

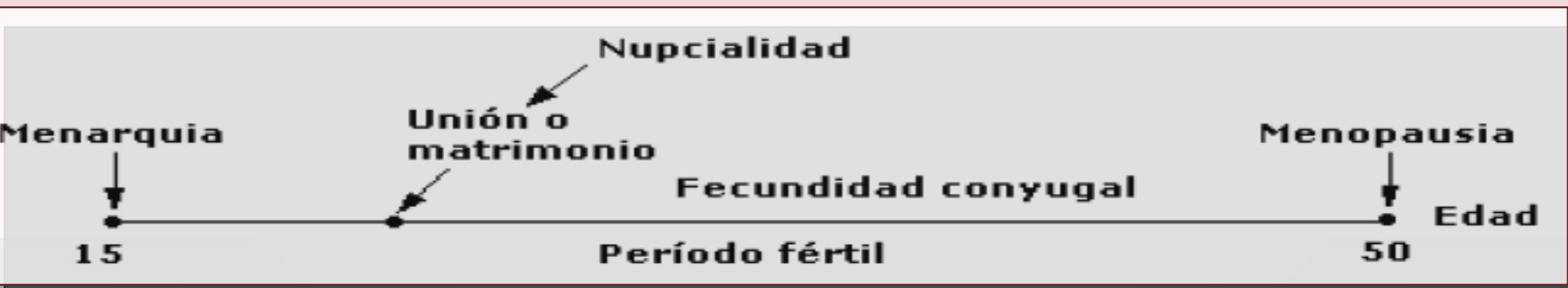




\section{Fuentes de información}

- Las estadísticas vitales

- Los Censos de población

- Encuestas demográficas

Las principales TASAS DE FECUNDIDAD utilizadas son:

- TASA DE FECUNDIDAD GENERAL (TFG),

- TASA DE FECUNDIDAD POR EDADES (TFE),

- INDICE SINTÉTICO DE FECUNDIDAD (ISF)o TASA GLOBAL DE FECUNDIDAD (TGF) o número de HIJOS por mujer,

- TASA BRUTA DE REPRODUCCIÓN (TBR o R')

- TASA NETA DE REPRODUCCIÓN (TNR o R) o número de HIJAS por mujer. 


\section{TASA DE FECUNDIDAD GENERAL (TFG)}

- La Tasa de Fecundidad General, que es la razón entre el número de nacidos vivos en un período de tiempo concreto (normalmente un año) relacionado con la cantidad de mujeres en edad fértil (entre 15 y 49 años) que existen durante ese período.

(TFG) Tasa de $=\quad \mathbf{N}^{\circ}$ de Nacidos (en el período) $\times 1000$ Fecundidad $N^{\circ}$ de mujeres (15-49 años)

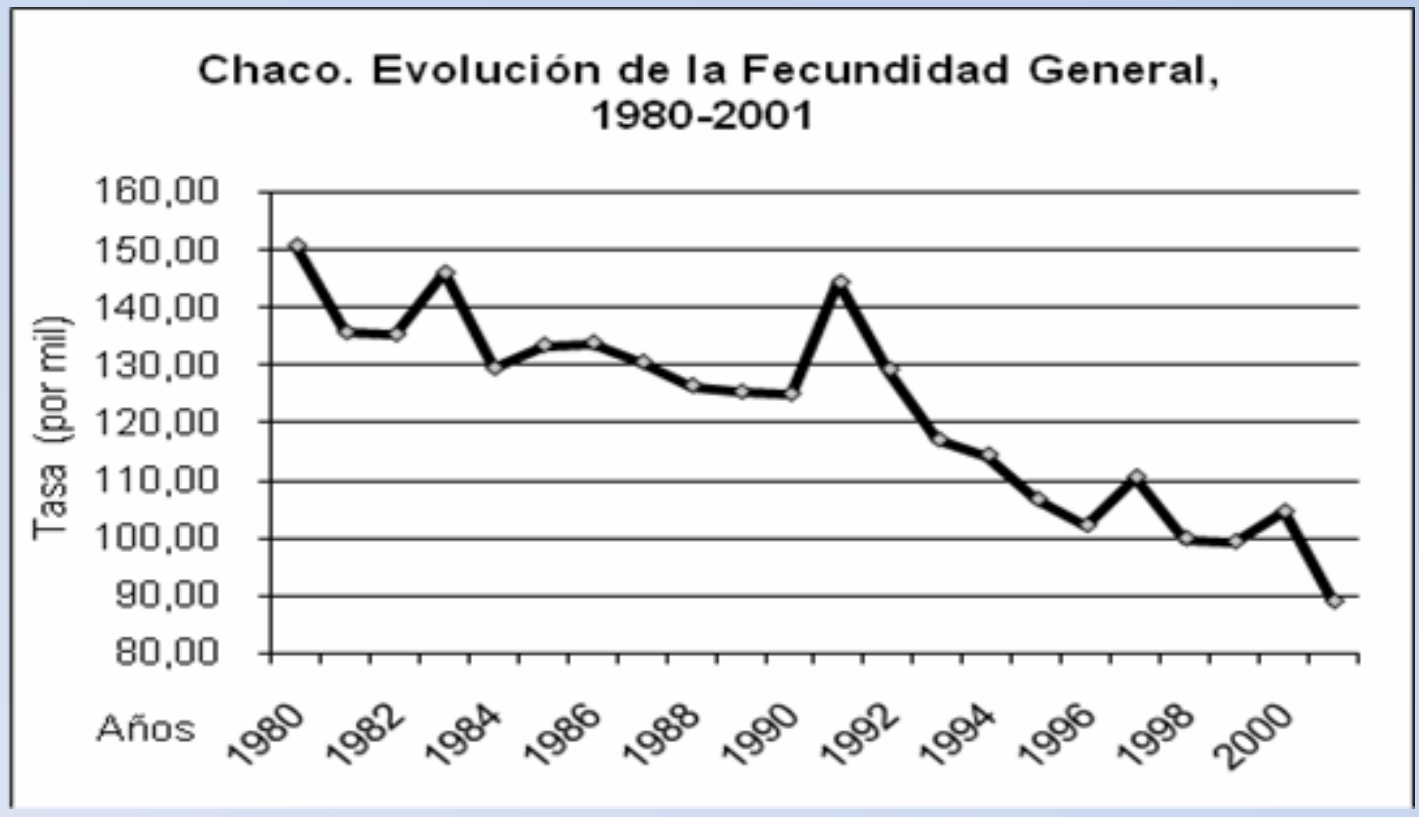

Publicado en formato digital: Foschiatti, Ana María. LA NATALIDAD y LA FECUNDIDAD. Resúmenes. Revista Geográfica Digital. IGUNNE. Facultad de Humanidades. UNNE. Año 7. № 13. Enero - Junio 2010. Resistencia, Chaco. En: http://hum.unne.edu.ar/revistas/geoweb/default.htm 
Representan la frecuencia con que ocurren los nacimientos en una determinada edad o grupo de edades, con respecto a la población femenina en una edad o grupo.

$$
\begin{aligned}
& f_{x}=\frac{B x(t, t+k)}{\operatorname{Pfx}(t, t+k)} * 1000 \\
& { }_{n} f_{x}=\frac{n B x(t, t+k)}{n \operatorname{Pfx}(t, t+k)} * 1000
\end{aligned}
$$




\section{La representación gráfica de estas tasas es la siguiente:}

\begin{tabular}{|c|c|c|c|c|c|}
\hline Edad & Nacimientos & & $\begin{array}{c}\text { Nacimientos } \\
\text { corregidos }\end{array}$ & $\begin{array}{l}\text { Población } \\
\text { femenina }\end{array}$ & $\begin{array}{c}\text { Tasas de } \\
\text { fecundidad } \\
\text { (por mil mujeres) }\end{array}$ \\
\hline Menos del5 & 611 & & - & - & - \\
\hline $15-19$ & 15999 & & 16610 & 193502 & 86 \\
\hline $20-24$ & 22565 & & 22565 & 171049 & 132 \\
\hline $25-29$ & 17902 & & 17902 & 149345 & 120 \\
\hline $30-34$ & 12490 & & 12490 & $15036 \mid$ & 83 \\
\hline $35-39$ & 6534 & & 6534 & 147652 & 44 \\
\hline $40-44$ & 1675 & & 1675 & 122409 & 14 \\
\hline $45-49$ & 118 & & 123 & 93306 & I \\
\hline 50 y más & 5 & & - & - & - \\
\hline Total & 77899 & & 77899 & I 027624 & 480 \\
\hline & & 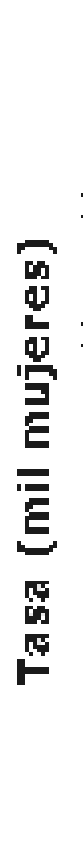 & $\begin{array}{l}\text { Costa } \\
60] \\
20] \\
00] \\
10\end{array}$ & $\begin{array}{l}30 \\
\text { Edad }\end{array}$ & $\begin{array}{l}\text { ficas de } \\
00\end{array}$ \\
\hline
\end{tabular}




\section{TIPOS DE ESTRUCTURA DE FECUNDIDAD POR EDADES}

Se definen varias curvas básicas que muestran el patrón de la fecundidad de una población:

- Cúspide temprana: La máxima fecundidad se presenta en el grupo de edades 20-24 años

- Cúspide dilatada: La máxima fecundidad se presenta entre los dos grupos de edades 20-24 y 25-29.

- Cúspide tardía: La máxima fecundidad se presenta en el grupo de edades 25-29 años.

- Cúspide prolongada: La máxima fecundidad se presenta entre los grupos de edades de 25-29 y 30-34.

- Cúspide extensa: Las edades con máximos valores se extienden por varios intervalos, incluso desde las edades jóvenes (20-24 a 30-34) 


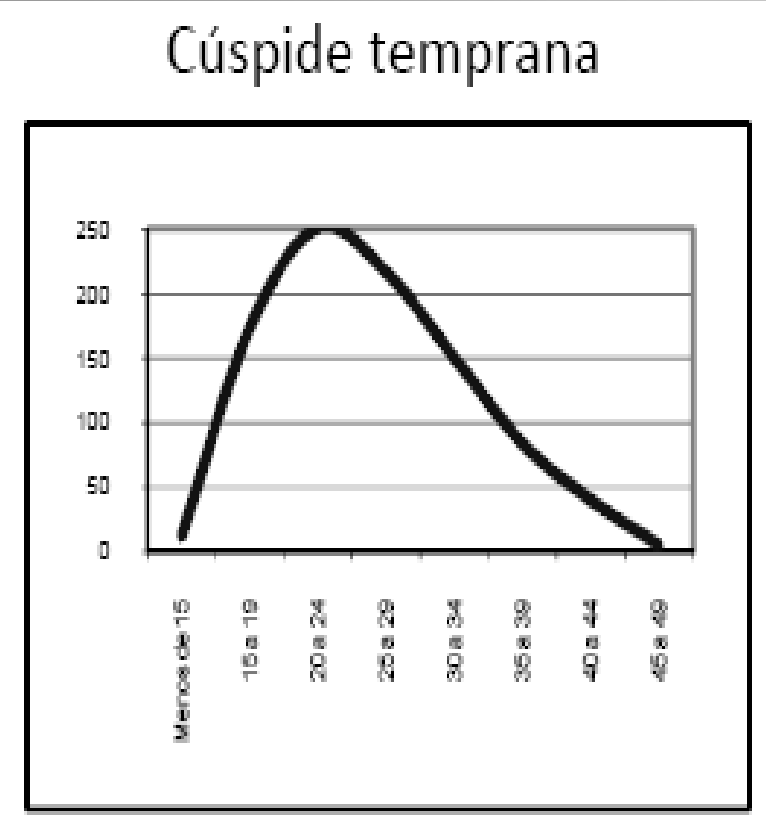

Cúspide tardía

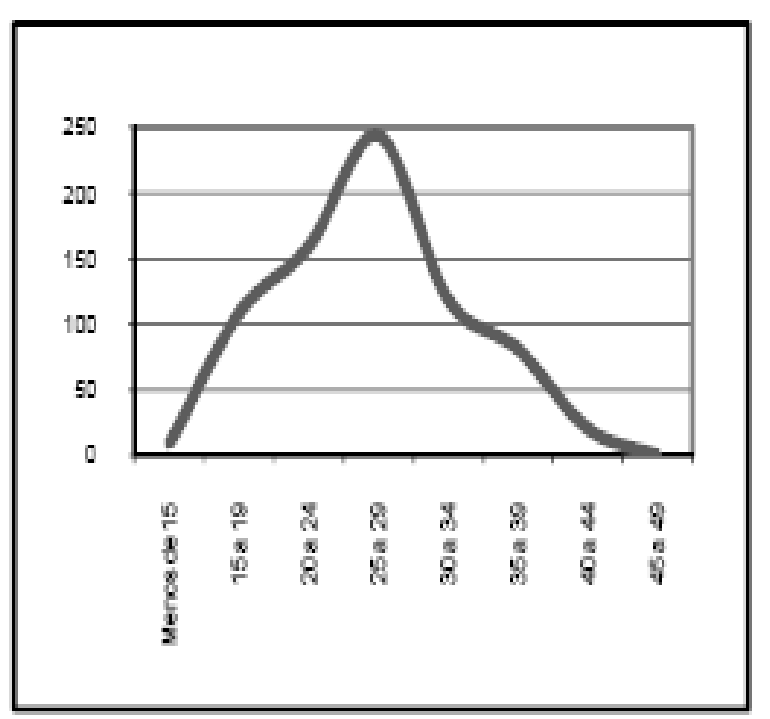

Cúspide dilatada

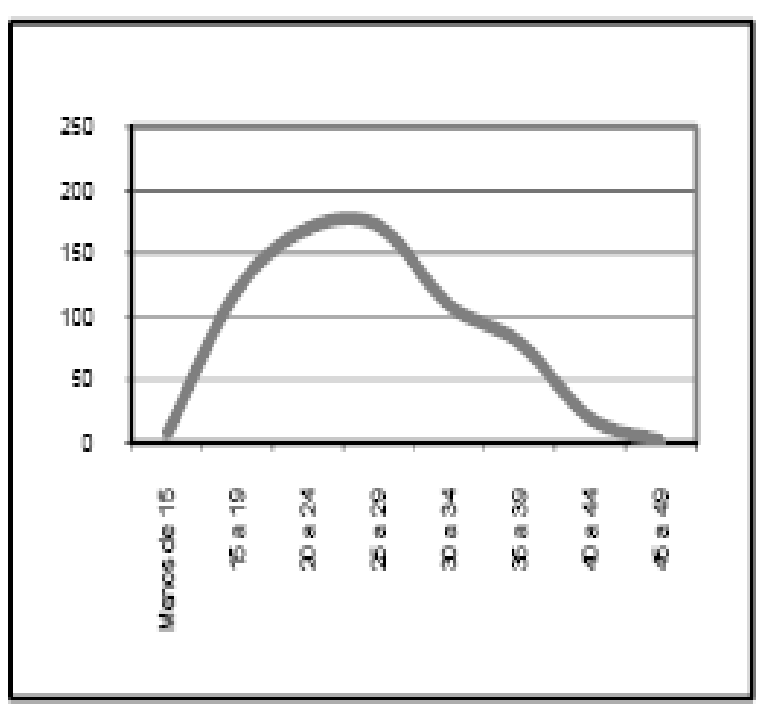

Cúspide prolongada

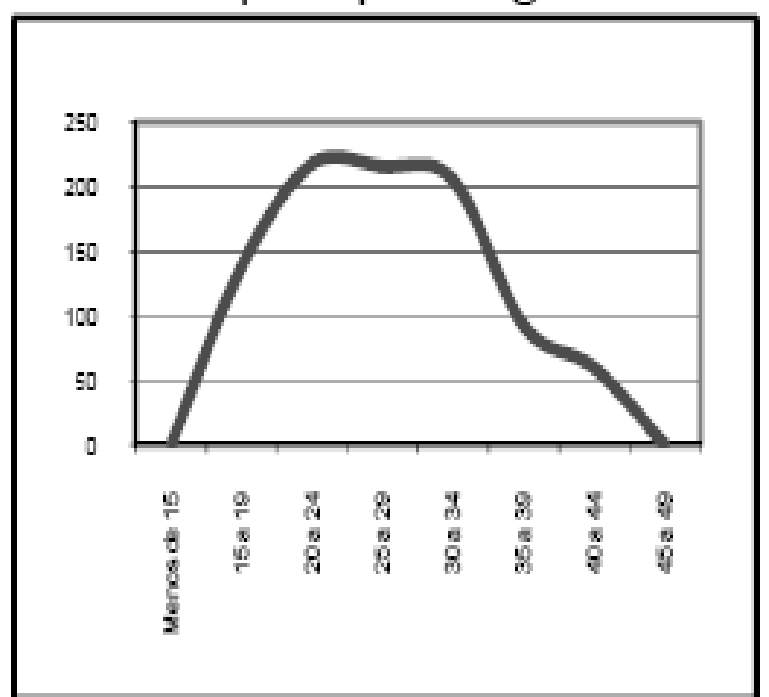

Cúspide extensa

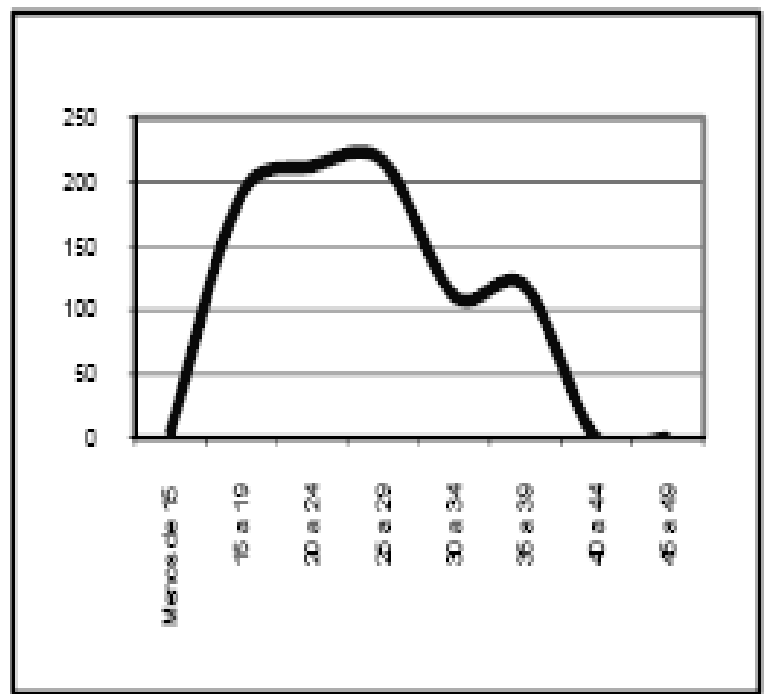

Publicado en formato digital: Foschiatti, Ana María. LA NATALIDAD y LA FECUNDIDAD. Resúmenes. Revista Geográfica Digital. IGUNNE. Facultad de Humanidades. UNNE. Año 7. № 13. Enero - Junio 2010. Resistencia, Chaco. En: http://hum.unne.edu.ar/revistas/geoweb/default.htm 


\section{Edad Media de la Fecundidad (EMF)}

- La edad media de la fecundidad o edad

esperada representa la edad a la que en promedio tienen sus hijos las mujeres.

- Estas tasas representan la frecuencia con que ocurren los nacimientos para una determinada edad o grupo de edades, con respecto a la cantidad de mujeres de ese grupo. 


\section{INDICE SINTÉTICO DE FECUNDIDAD O TASA GLOBAL DE}

\section{FECUNDIDAD (ISF O TGF)}

- Esta es una de las principales medidas de fecundidad. Es utilizada mundialmente para realizar comparaciones en los patrones de fecundidad, entre las diferentes poblaciones.

- La TGF o Índice sintético se obtiene de la suma de las tasas específicas de fecundidad por edades multiplicado por el intervalo en el que éstas se han agrupado (normalmente el intervalo es 5 , es decir de $n$ veces), cuando se trabaja con grupos de edades entre " $x$ " $y$ " $x+n$ ". Es decir:

$$
\text { ISF ó TGF }=\sum_{x} f_{x} \quad \text { ISF ó TGF }=\sum_{x} * \sum_{n} f_{x} * 1000
$$

\section{NÚMERO DE HIJOS POR MUJER}

$\mathrm{ISF}=\underline{\mathrm{TFG} * 35}$

\section{0}

(número de hijos que una mujer podría tener durante su vida fértil) 


\section{TASAS DE REPRODUCCIÓN O REEMPLAZO}

- Tasa bruta de reproducción (TBR)

$\mathrm{TBR}=\underline{\underline{100}} * \mathrm{TGF}=\mathbf{0 , 4 8 7 8} * \mathrm{TGF}$ 205

- Tasa neta de reproducción (TNR)

$$
\mathrm{TNR}=\frac{100}{205} * \mathrm{TGF}_{*} \mathrm{PF}_{\mathbf{0}}=\mathbf{0 , 4 8 7 8} * \mathrm{TGF}_{*} \mathrm{PF}_{\mathbf{0}}
$$

- Relación Niños - Mujer (RNM)

$$
\text { RNM }=\frac{5^{\mathrm{N}} 0}{35^{\mathrm{NF}} 15} \text {. }
$$




\section{FACTORES QUE INFLUYEN EN LA FECUNDIDAD}

- Factores fisiológicos: factores de comportamiento, frecuencia de las relaciones, período de lactancia, calidad de la alimentación, a los métodos anticonceptivos

- Factores demográficos: proporción de mujeres en edad fértil, duración media de los matrimonios y edad de las uniones.

- Factores sociales: emancipación de la mujer, educación femenina, clase social, nupcialidad, tamaño familiar y desarrollo cultural.

- Factores económicos: incorporación de la mujer al mundo laboral, tipo de actividad económica, rol económico de los hijos.

- Factores geográficos: fecundidad en el ámbito urbano y rural.

- Factores políticos: políticas gubernamentales pro y antinatalista, control de nacimientos, desarrollo de la planificación familiar, prohibición del trabajo infantil, educación obligatoria, aborto, ayudas económicas.

- Factores psicológicos: perspectivas laborales, percepción de la posibilidad de ajustar la vida laboral con la vida familiar. 


\section{NIVELES MUNDIALES DE FECUNDIDAD}

Para el conjunto del mundo el ISF es de 3.6 hijos/mujer, oscilando entre valores tan extremos como 8.5 en Ruanda y 8.1 en Kenia y 1.4 en Austria, República Federal de Alemania y Luxemburgo o 1.3 en Italia y Hong Kong.

Se destacan:

a) Países con muy alta fecundidad (más de 5 hijos/mujer)

b) Países de alta fecundidad (3,6-5 hijos/mujer).

c) Países de fecundidad moderada (2.1-3.5 hijos/mujer).

d) Países de baja fecundidad (con 2 ó menos hijos/mujer). 


\section{Se destacan tres etapas}

- Una primera, entre 1950-55 y 1960-65: existencia de dos grupos plenamente diferenciados en sus tasas:

las regiones en desarrollo, con un índice superior a 5 hijos por mujer (media mundial), que comprenden Asia y América Latina (con cifras similares y cercanas a 6) y África, con un promedio de 7 hijos/mujer las regiones desarrolladas, con índices que oscilan entre 2,5 y 4 hijos/ mujer, agrupando los países de Europa y América del Norte que más pronto completaron su transición demográfica desde altas a bajas tasas. 
- Una segunda etapa, entre 1960-65 y 1985-90

Descenso sistemático de la tasa en el conjunto mundial pero con mayor relevancia en las regiones en desarrollo de Asia y América Latina, (3.5 hijos/mujer), América del Norte, que desde 1970 sitúa su índice por debajo del mínimo de sustitución generacional, y Oceanía, con niveles reproductivos superiores a 2,5 hijos/mujer.

África muestra un descenso lento en la reducción de su fecundidad manteniéndola por encima de 6 hijos/mujer;

Europa con una suave pendiente, a pesar de lo cual registraba ya en 1975-80 un índice de 2 hijos/mujer;

y la U.R.S.S. se caracteriza por ligeras oscilaciones, en torno a 2.32.4 hijos/mujer. 
- Una tercera fase futura, entre 1985-90 y 2020-25

Sustancial reducción de la fecundidad en el continente africano (de 6.3 a 3.0 hijos/mujer),

Decremento también en América Latina que alcanzaría al final del período niveles inferiores a 2.5 hijos/mujer y, sobre todo, Asia que llegaría a registrar en el 2025 un valor escasamente superior a $2 \mathrm{Hijos} /$ mujer e inferior incluso a los niveles de Oceanía o la U.R.S.S.

En Europa y América del Norte se prevé un ligero y lento ascenso de las tasas que, sin embargo, no llegarían a implicar valores por encima del mínimo necesario para el reemplazo generacional 


\section{NUPCIALIDAD}

La nupcialidad se refiere al matrimonio como un fenómeno poblacional, incluyendo su cuantificación, las características de las personas unidas en matrimonio y la disolución de esas uniones (mediante el divorcio, la separación, la viudez y la anulación).

- TASA DE NUPCIALIDAD. (o Tasa bruta de nupcialidad) es el número de matrimonios por 1000 personas en un lapso determinado (generalmente un año). Esta tasa se calcula utilizando el número de matrimonios - no el número de personas que se casan - e incluye tanto las primeras como las segundas nupcias.

\section{Número de matrimonios}

\section{Población total}

\subsection{3}

$\times 1000=$

2.308.300 